\title{
Second Guessing in Perceptual Decision-Making
}

\author{
Charlotte S. McLean, ${ }^{\star}{ }^{\circledR}$ Bowen Ouyang, ${ }^{\star}$ and ${ }^{\circledR}$ Jochen Ditterich \\ Center for Neuroscience and Department of Neurobiology, Physiology \& Behavior, University of California at Davis, Davis, California 95618
}

Human subjects of both sexes were asked to make a perceptual decision between multiple directions of visual motion. In addition to reporting a primary choice, they also had to report a second guess, indicating which of the remaining options they would rather bet on, assuming that they got their primary choice wrong. The second guess was clearly informed by the amounts of sensory evidence that were provided for the different options. A single computational integration-to-threshold model, based on the assumption that the second guess is determined by the rank ordering of accumulated evidence at or shortly after the time of the decision, was able to explain the distribution of primary choices, associated response times, and the distribution of second guesses. This suggests that the decision-maker has access to how well supported unchosen options are by the sensory evidence.

Key words: accumulated evidence; computational model; multiple alternatives; perceptual decision; second choice; second guess

\section{Significance Statement}

Perceptual decisions require conversion of sensory evidence into a discrete choice. Computational models based on the accumulation of evidence to a decision threshold can explain the distribution of choices and associated decision times. Subjects are also able to report the level of confidence in their decision. Here we show that, when making decisions between more than two alternatives, the decision-maker can even report a second guess that is clearly informed by the sensory evidence. These second guesses show a distribution that is consistent with subjects having access to how much sensory evidence was accumulated for the unchosen options. The decision-maker therefore has knowledge about the outcome of the decision process that goes beyond just the choice and an associated confidence.

\section{Introduction}

Perceptual decisions require a decision-maker to make a discrete choice on the basis of sensory information. Substantial work has gone into elucidating the mechanisms that allow the inflowing sensory evidence to be converted into a discrete choice. Integration-to-threshold mechanisms are the currently dominant class of models, the Drift Diffusion Model being a popular exemplar (Luce, 1986; Ditterich, 2006, 2010; Ratcliff and McKoon, 2008; Forstmann et al., 2016). These models are based on the idea that sensory evidence for each available option is accumulated until the accumulated evidence for one of the options exceeds a decision threshold. They can explain the distribution of choices and associated response times (RTs) for a wide range of decision tasks (Ratcliff and Smith, 2004) and are consistent

\footnotetext{
Received Nov. 22, 2019; revised May 5, 2020; accepted May 7, 2020.

Author contributions: C.S.M. and B.O. performed research; J.D. designed research; J.D. analyzed data; J.D. wrote the paper.

This work was supported by National Science Foundation Grant 1156601.

The authors declare no competing financial interests.

*C.S.M. and B.O. contributed equally to this work.

C.S. McLean's present address: University of Texas Southwestern Medical Center, Dallas, TX 75390.

Correspondence should be addressed to Jochen Ditterich at jditterich@ucdavis.edu.

https://doi.org/10.1523/JNEUROSCI.2787-19.2020

Copyright $\odot 2020$ the authors
}

with decision-related neural activity, both averaged across trials as well as on a single-trial level (Ditterich, 2006; Bollimunta et al., 2012). It is difficult, however, to pinpoint experimentally how much temporal integration is involved in the process (Ditterich, 2006), and the view that single-trial decision-related neural activity is consistent with a diffusion-like process has been challenged (Latimer et al., 2015).

More recently, confidence in a perceptual decision has become the focus of scientific investigation. Some studies suggest that a common mechanism could explain both the outcome of the decision as well as the reported confidence (Kiani and Shadlen, 2009; Kiani et al., 2014), while other reports have focused on dissociations between subjective confidence and objective decision accuracy (for review, see Rahnev and Denison, 2018). When making binary decisions, the choice and the associated confidence fully describe the outcome of the decision process. When making decisions between more than two alternatives, the decision-maker could also have knowledge about how well the sensory evidence supported the unchosen options.

Here we ask whether human subjects have access to information about the "relative desirability" of the unchosen options when making perceptual decisions between more than two alternatives and, if so, whether one can provide a quantitative explanation for the distribution of reported second guesses. We used a modified version of the 3 -alternative forced choice version of the 


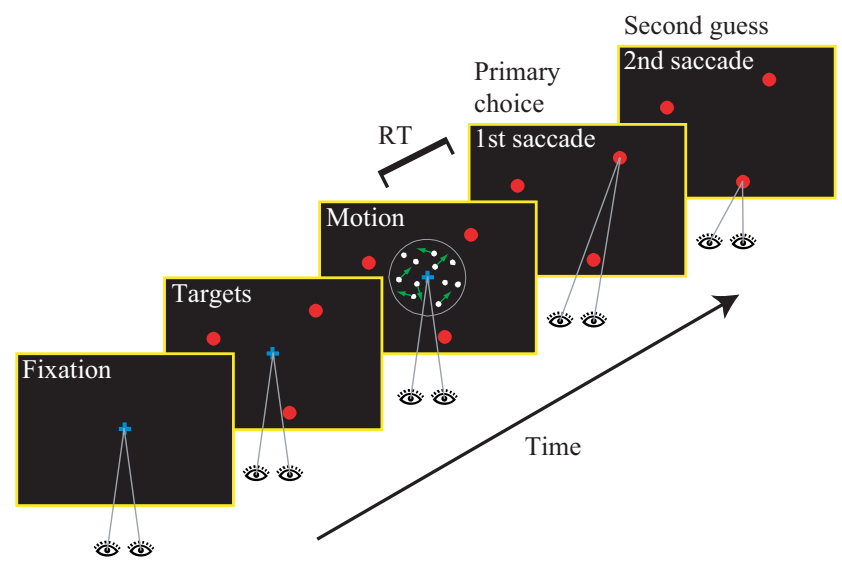

Figure 1. Experimental paradigm. Human subjects were asked to determine the strongest motion direction in a random-dot pattern with multiple motion components. They were free to watch the stimulus as long as they wanted and responded with a goal-directed eye movement to one of three choice targets to indicate their primary choice. Choices and RTs were measured. After indicating their primary choice, subjects were instructed to make a second goal-directed eye movement to one of the remaining two targets to indicate a second guess.

multicomponent random-dot motion direction discrimination task introduced by Niwa and Ditterich (2008). Briefly, the subject watches a random-dot motion stimulus that simultaneously contains coherent motion in three different directions, all separated by 120 degrees. The strength of each motion component is chosen randomly. The observer has to determine the strongest motion component and indicate its direction with an eye movement. The choice and the associated RT are recorded. For this study, subjects were instructed to also report a second guess with a second eye movement. We asked the observers to indicate which of the remaining two options they would rather bet on, assuming they got their primary choice wrong. Once both the primary choice and the second guess had been registered, auditory feedback about the accuracy of the primary choice was provided. Subjects did not receive feedback on their second guess. The task is illustrated in Figure 1. Further details regarding the experimental design can be found in Materials and Methods.

Here we demonstrate that the second guess is clearly informed by the sensory evidence and that a single integrationto-threshold model can explain the distribution of primary choices, associated RTs, and the distribution of second guesses. This suggests that the decision-maker has access to how much sensory evidence had been accumulated for options other than the chosen one at the time when the decision was made. We also consider alternative models and show that the second-best explanation for the data are provided by a model that starts a new decision process between the remaining alternatives when the primary decision is made and reads out the decision variable after a fixed amount of time.

\section{Materials and Methods}

Experimental design and statistical analyses

Human subjects. The study was approved by the University of California at Davis Institutional Review Board. After giving their informed consent, 7 University of California at Davis undergraduate students (4 females, 3 males) with normal or corrected-to-normal vision participated in the experiment. Each of the subjects completed at least five experimental sessions with a minimum of 300 valid decision trials each.

Experimental setup. The subjects sat in front of a 22 inch flat-screen CRT video monitor (ViewSonic P225f; viewing distance: $60 \mathrm{~cm}$ ) with their head on a chin and forehead rest. The visual stimuli were generated by a Macintosh G4 computer running Mac OS 9, MATLAB (The MathWorks), and the Psychophysics Toolbox (Brainard, 1997; Pelli, 1997) at a frame rate of $75 \mathrm{~Hz}$. The experiment was controlled and the data were collected by an Intel Pentium IV computer running QNX (Ottawa) and a modified version of REX (Laboratory of Sensorimotor Research, National Eye Institute).

Eye movements were monitored using a monocular IR video eye tracker with chinrest-mounted optics (Series 5000, Applied Science Laboratories) operating at $240 \mathrm{~Hz}$. Before each experimental session, the eye tracker was calibrated using repeated fixation of nine calibration targets with horizontal eccentricities of $-10,0$, and 10 degrees and vertical eccentricities of $-7.5,0$, and 7.5 degrees.

Experimental task and visual stimulus. The experimental task is illustrated in Figure 1. Each trial started with the presentation of a central fixation mark (diameter: 0.3 degree). The measured fixation location had to remain within 2.5 degrees of the center of the screen throughout the trial (up to the saccadic response). After $250-500 \mathrm{~ms}$ of stable fixation, three targets (diameter: 0.5 degree) appeared on the screen, all located on a virtual circle around the fixation mark with a radius of 8.0 degrees. The target locations were chosen randomly (with equal spacing) at the beginning of an experimental session and did not change throughout the session. After another random delay of $250-500 \mathrm{~ms}$, a multicomponent random-dot pattern was presented at the center of the screen (diameter: 5.0 degrees).

In the original version of the stimulus (as used, e.g., by Shadlen and Newsome, 2001; Roitman and Shadlen, 2002; Palmer et al., 2005) a certain fraction of the dots (defined as the coherence of the stimulus) was moving coherently in a particular direction, whereas the remaining dots were flickering randomly. Our multicomponent random-dot pattern had up to three coherent motion components embedded. Thus, there were four subpopulations of dots: one was moving coherently in a particular direction $\theta$ (aligned with one of the choice targets; fraction of dots defined by the coherence of the first component), another one was moving coherently in the direction $\theta+120^{\circ}$ (fraction defined by the coherence of the second component), a third one was moving coherently in the direction $\theta+240^{\circ}$ (fraction defined by the coherence of the third component), and the remaining dots were flickering randomly. The stimulus is therefore described by a set of three coherences. Which of the four subpopulations a particular dot belonged to changed randomly over time. As a consequence, the stimulus is not perceived as an overlay of several transparent layers of motion that could be easily separated, but as a mixture of different motion components. For a discussion of transparent random-dot motion stimuli, see, for example, Treue et al. (2000). Corresponding pairs of dots, responsible for the percept of apparent motion, were presented with a temporal separation of $40 \mathrm{~ms}$ (three video frames). The coherently moving dots had a speed of $6 \mathrm{deg} / \mathrm{s}$, the dot density was $16.7 \frac{d o t s}{\operatorname{deg}^{2} \cdot s^{\prime}}$, and each dot was a little filled square with an edge length of 0.1 degree. On each trial, the set of coherences was randomly selected from a list of 51 possible coherence combinations ranging from $0 \%$ to $40 \%$ each. The full list can be found in Table 1 .

The subjects were instructed to identify the direction of the strongest motion component and to make a saccadic eye movement to the associated choice target (aligned with the identified direction of motion). They were allowed to watch the stimulus for as long as they wanted (up to $5 \mathrm{~s}$ ) and to respond whenever they were ready. The motion stimulus disappeared from the screen as soon as the eye left the central fixation window. Subjects were further instructed to indicate with a second saccadic eye movement to one of the two remaining choice targets, which of the remaining options they would rather bet on as a second guess, assuming they got their first choice wrong. After each trial, they received auditory feedback as to whether they had picked the correct target in their primary choice. In case the stimulus did not have one strongest motion component, the computer randomly identified one of the targets as being the correct one. No feedback was given on the second guess.

In order to complete a trial successfully ("valid trial"), the subject had to maintain accurate fixation until the random-dot pattern appeared. Once central fixation was broken, the eye position had to be within 3 degrees of one of the three choice targets within $100 \mathrm{~ms}$ and had to stay 
Table 1. List of motion coherence combinations

\begin{tabular}{|c|c|c|}
\hline $\begin{array}{l}\text { Motion coherence of } \\
\text { first component (\%) }\end{array}$ & $\begin{array}{l}\text { Motion coherence of } \\
\text { second component (\%) }\end{array}$ & $\begin{array}{l}\text { Motion coherence of } \\
\text { third component (\%) }\end{array}$ \\
\hline 0 & 0 & 0 \\
\hline 5 & 0 & 0 \\
\hline 0 & 5 & 0 \\
\hline 0 & 0 & 5 \\
\hline 10 & 0 & 0 \\
\hline 0 & 10 & 0 \\
\hline 0 & 0 & 10 \\
\hline 20 & 0 & 0 \\
\hline 0 & 20 & 0 \\
\hline 0 & 0 & 20 \\
\hline 40 & 0 & 0 \\
\hline 0 & 40 & 0 \\
\hline 0 & 0 & 40 \\
\hline 10 & 10 & 10 \\
\hline 20 & 10 & 10 \\
\hline 10 & 20 & 10 \\
\hline 10 & 10 & 20 \\
\hline 30 & 10 & 10 \\
\hline 10 & 30 & 10 \\
\hline 10 & 10 & 30 \\
\hline 20 & 15 & 5 \\
\hline 20 & 5 & 15 \\
\hline 15 & 20 & 5 \\
\hline 5 & 20 & 15 \\
\hline 15 & 5 & 20 \\
\hline 5 & 15 & 20 \\
\hline 30 & 15 & 5 \\
\hline 30 & 5 & 15 \\
\hline 15 & 30 & 5 \\
\hline 5 & 30 & 15 \\
\hline 15 & 5 & 30 \\
\hline 5 & 15 & 30 \\
\hline 20 & 20 & 20 \\
\hline 30 & 20 & 20 \\
\hline 20 & 30 & 20 \\
\hline 20 & 20 & 30 \\
\hline 40 & 20 & 20 \\
\hline 20 & 40 & 20 \\
\hline 20 & 20 & 40 \\
\hline 30 & 25 & 15 \\
\hline 30 & 15 & 25 \\
\hline 25 & 30 & 15 \\
\hline 15 & 30 & 25 \\
\hline 25 & 15 & 30 \\
\hline 15 & 25 & 30 \\
\hline 40 & 25 & 15 \\
\hline 40 & 15 & 25 \\
\hline 25 & 40 & 15 \\
\hline 15 & 40 & 25 \\
\hline 25 & 15 & 40 \\
\hline 15 & 25 & 40 \\
\hline
\end{tabular}

on this target (primary choice) for at least $200 \mathrm{~ms}$. At this point, a neutral sound was played, indicating that the primary choice had been registered, but not providing any information about its accuracy yet. At most 3 s later, the eye position had to be within 3 degrees of one of the remaining choice targets and had to stay on this target (second guess) for at least $200 \mathrm{~ms}$. At this point, auditory feedback was given about the accuracy of the primary choice, which indicated to the subject that the trial had been registered as a valid trial.

Data analysis. For analyzing the data, we collapsed across different target locations. Thus, we only worked with the 15 unique sets of coherences (eliminating the permutations) and whether the subject picked the target associated with the strongest motion component, the one associated with the intermediate component, or the one associated with the weakest component. We analyzed the pooled data across subjects to have a robust number of trials for each experimental condition. Since we only work with mean RTs in this study, we were not concerned about variability in RT across subjects potentially affecting the shape of RT distributions.

RT was defined as the time between the appearance of the randomdot stimulus and the breaking of central fixation. We did not analyze the timing of the second guess as subjects had to wait for their primary choice to be registered by the computer before they could report their second guess. Thus, the timing of the second guess was largely externally imposed.

\section{Computational models}

Model of the neural representation of the sensory stimulus. The mean response of a population of motion-sensitive neurons to a 3-component random-dot stimulus with coherences $c_{1}$ (in the preferred direction of the pool), $c_{2}$, and $c_{3}$ was modeled to be of the following form:

$$
\overline{s_{1}}=\frac{g \cdot\left[c_{1}+k_{n} \cdot\left(1-\sum_{i=1}^{3} c_{i}\right)\right]}{1+k_{s} \cdot\left(c_{2}+c_{3}\right)}
$$

where $g$ is the overall gain of the sensory response (relationship between neural activity and motion strength). The two additive terms in the brackets reflect the two linear response components: the first describes the response to the coherent motion in the preferred direction, and the second describes the response to the noise dots. The term in parentheses reflects the proportion of noise dots in the stimulus. $k_{n}$ is the relative gain of the response to the noise dots compared with the response to an identical fraction of dots moving coherently in the preferred direction. The term in the denominator reflects the divisive normalization. Since the term in the numerator accurately describes the response to a singlecomponent stimulus, only the coherences of motion components with directions other than the preferred one are present in the denominator. For simplicity, we have chosen a linear term, with $k_{s}$ describing the gain/ strength of the divisive normalization (Niwa and Ditterich, 2008).

In general, the mean responses of each of the three task-relevant sensory pools can be written as follows:

$$
\overline{s_{j}}=\frac{g \cdot\left[c_{j}+k_{n} \cdot\left(1-\sum_{i=1}^{3} c_{i}\right)\right]}{1+k_{s} \cdot \sum_{i \neq j} c_{i}}
$$

The variances of the three sensory responses were modeled as follows:

$$
\sigma_{s_{j}}^{2}=k_{v} \cdot \overline{s_{j}}
$$

We described the outputs of the sensory pools as normal random processes to be able to treat the decision process as a standard diffusion process (based on Brownian motion), which is reasonable if the pools are not too small.

Model of the decision process. In principle, we would have to treat the race between the three integrators mathematically as a $3 \mathrm{D}$ diffusion process. However, for the 2-alternative forced choice (2AFC) case, the decision process has often been described as a $1 \mathrm{D}$ diffusion process with two boundaries instead of a $2 \mathrm{D}$ diffusion process. This simplification can be done when one assumes that the two signals that are accumulated by the two integrators are only different in sign, but identical in absolute value. Such a situation would result from all of the contributions that a particular pool of sensory neurons makes to the net evidence signals having the same origin. If we make the same assumption in our model, we can also reduce the dimensionality of the problem. We can write the three evidence signals as follows: 


$$
\begin{aligned}
& e_{1}=s_{1}-\frac{1}{2} s_{2}-\frac{1}{2} s_{3} \\
& e_{2}=s_{2}-\frac{1}{2} s_{1}-\frac{1}{2} s_{3} \\
& e_{3}=s_{3}-\frac{1}{2} s_{1}-\frac{1}{2} s_{2}
\end{aligned}
$$

$e_{3}$ can be rewritten as follows:

$$
e_{3}=-\left(s_{1}-\frac{1}{2} s_{2}-\frac{1}{2} s_{3}\right)-\left(s_{2}-\frac{1}{2} s_{1}-\frac{1}{2} s_{3}\right)=-e_{1}-e_{2}
$$

Thus, if $e_{1}$ and $e_{2}$ are known, $e_{3}$ is known. In our model, each of the three evidence signals is integrated over time (Fig. 2) as follows:

$$
i_{j}(t)=\int_{0}^{t} e_{j}(\tau) d \tau
$$

Since integration is a linear operation, if $i_{1}$ and $i_{2}$ are known, $i_{3}$ is also known. We can therefore rewrite the decision criterion for choosing the third alternative as follows:

$$
\begin{array}{ccc}
i_{3} & > & 1 \\
-i_{1}-i_{2} & > & 1 \\
i_{2} & < & -i_{1}-1
\end{array}
$$

Thus, the third integrator exceeding a value of 1 is equivalent to crossing another linear boundary in the $i_{1}-i_{2}$ plane (for an illustration, see Niwa and Ditterich, 2008, their Fig. 3C). The diffusion process always starts at $(0 ; 0)$ and stops when one of the three boundaries is crossed: $i_{1}=$ 1 is the decision boundary for the first alternative, $i_{2}=1$ is the boundary for the second alternative, and $i_{2}=-i_{1}-1$ is the boundary for the third alternative.

The $2 \mathrm{D}$ diffusion process is described by a drift vector and a covariance matrix. The drift vector is given by $\left[\overline{\frac{e_{1}}{e_{2}}}\right]$, the means of the first two evidence signals. Since $\left[\begin{array}{l}e_{1} \\ e_{2}\end{array}\right]$ can be calculated as follows:

$$
\left[\begin{array}{l}
e_{1} \\
e_{2}
\end{array}\right]=\left[\begin{array}{ccc}
1 & -\frac{1}{2} & -\frac{1}{2} \\
-\frac{1}{2} & 1 & -\frac{1}{2}
\end{array}\right] \cdot\left[\begin{array}{l}
s_{1} \\
s_{2} \\
s_{3}
\end{array}\right]
$$

$\left[\begin{array}{l}\overline{e_{1}} \\ \overline{e_{2}}\end{array}\right]$ is given by the following:

$$
\left[\begin{array}{l}
\overline{e_{1}} \\
\overline{e_{2}}
\end{array}\right]=\left[\begin{array}{ccc}
1 & -\frac{1}{2} & -\frac{1}{2} \\
-\frac{1}{2} & 1 & -\frac{1}{2}
\end{array}\right] \cdot\left[\begin{array}{c}
\overline{s_{1}} \\
\overline{s_{2}} \\
\overline{s_{3}}
\end{array}\right]
$$

The covariance matrix $\Sigma$ can be calculated as follows:

$$
\begin{aligned}
& \Sigma=\left[\begin{array}{ccc}
1 & -\frac{1}{2} & -\frac{1}{2} \\
-\frac{1}{2} & 1 & -\frac{1}{2}
\end{array}\right] \cdot\left[\begin{array}{ccc}
\sigma_{s_{1}}^{2} & 0 & 0 \\
0 & \sigma_{s_{2}}^{2} & 0 \\
0 & 0 & \sigma_{s_{3}}^{2}
\end{array}\right] \cdot\left[\begin{array}{cc}
1 & -\frac{1}{2} \\
-\frac{1}{2} & 1 \\
-\frac{1}{2} & -\frac{1}{2}
\end{array}\right]= \\
& =\left[\begin{array}{cc}
\sigma_{s_{1}}^{2}+\frac{1}{4} \sigma_{s_{2}}^{2}+\frac{1}{4} \sigma_{s_{3}}^{2} & -\frac{1}{2} \sigma_{s_{1}}^{2}-\frac{1}{2} \sigma_{s_{2}}^{2}+\frac{1}{4} \sigma_{s_{3}}^{2} \\
-\frac{1}{2} \bar{\sigma}_{s_{1}}^{2}-\frac{1}{2} \overline{\sigma_{s_{2}}^{2}}+\frac{1}{4} \bar{\sigma}_{s_{3}}^{2} & \frac{1}{4} \sigma_{s_{1}}^{2}+\overline{\sigma_{s_{2}}^{2}}+\frac{1}{4} \sigma_{s_{3}}^{2}
\end{array}\right]= \\
& =k_{v} \cdot\left[\begin{array}{cc}
\overline{s_{1}}+\frac{1}{4} \overline{s_{2}}+\frac{1}{4} \overline{s_{3}} & -\frac{1}{2} \overline{s_{1}}-\frac{1}{2} \overline{s_{2}}+\frac{1}{4} \overline{s_{3}} \\
-\frac{1}{2} \overline{s_{1}}-\frac{1}{2} \overline{s_{2}}+\frac{1}{4} \overline{s_{3}} & \frac{1}{4} \overline{s_{1}}+\overline{s_{2}}+\frac{1}{4} \overline{s_{3}}
\end{array}\right]
\end{aligned}
$$

Model of the second choice based on accumulated evidence at or shortly after the time of the first threshold crossing. The integrator crossing the decision threshold first determines the primary choice and the decision time. We propose that the state of the remaining integrators at the time when the winning integrator crosses threshold can be used to determine the second guess. The higher value of the two remaining integrators determines the second choice. For example, if the second integrator crossed the threshold first, the states of the first and the third integrator at this particular time would be compared, and the larger value would determine the second guess. As pointed out above, when working with a $2 \mathrm{D}$ stochastic process, the two dimensions correspond to the states of the first two integrators. The state of the third integrator can be calculated as $i_{3}=-i_{1}-i_{2}$.

While the first passage time problem could be solved numerically (see Ditterich, 2006; Niwa and Ditterich, 2008), we also needed the predictions for the distribution of second choices. Therefore, we discretized the 2D diffusion process (time step: $5 \mathrm{~ms}$ ) and simulated 50,000 trials per experimental condition. The MATLAB function OU_2D_3B_ SIM_SC.M, which has been used for performing the model calculations, is part of the Stochastic Integration Modeling Toolbox (SIMT; written by J.D.), which can be downloaded from https://www.github.com/ peractionlab/StochInt.

To determine whether the second guess might have been informed by sensory evidence that arrived at the decision process after the decision threshold had been crossed, we allowed the integration process to continue for a fixed amount of time after the threshold crossing and then read out and compared the states of the integrators that had not won the race to threshold. The MATLAB function used for this purpose, OU_2D_3B_SIM_SC_ADD_TIME.M, is also part of SIMT. To quantify the deviation between predicted and observed second guesses, we calculated the sum of the squared differences between predicted and observed relative frequencies.

Model of the second choice based on two successive threshold crossings. We also considered a model where the integration process continues after the first threshold crossing, until a second (different) bound is crossed. The first threshold crossing determines the first choice, the second threshold crossing the second choice and the decision time. In contrast to our original model, to give this model more flexibility, the integration of sensory evidence was allowed to be leaky (the time constant of integration was an additional free model parameter), and the bounds were allowed to collapse over time. We used the same logistic function as in Ditterich (2006) as follows:

$$
A(t)=\frac{1}{1+\exp (s \cdot(t-d))}+\frac{\exp (-s \cdot d)}{1+\exp (-s \cdot d)}
$$

$t$ is the time into the decision process, and $s$ and $d$ are two additional free model parameters that define the shape (slope) and the position (delay) of the collapsing bound. The MATLAB function OU_2D_3B_ TWO_CROSS_SIM.M, which was used for evaluating this model, is also part of SIMT.

Models of the second choice based on two successive decision processes. Another class of models involved starting a new decision process when the first threshold crossing occurred, but only between the two alternatives that did not win the original race to threshold. For example, assuming that $i_{2}$ crossed the threshold first, the second process would be set up as follows:

$$
\begin{aligned}
& e_{1}^{\prime}=s_{1}-s_{3} \\
& e_{2}^{\prime}=s_{3}-s_{1}
\end{aligned}
$$

If the integral of the first evidence signal crossed the threshold first, Direction 1 would be reported as the second choice. If the integral of the second evidence signal crossed the threshold first, Direction 3 would be reported as the second choice. Since $e_{2}^{\prime}=-e_{1}^{\prime}$, this second decision process can be treated as a $1 \mathrm{D}$ drift-diffusion process with two boundaries. The decision time would be the total duration of both decision processes. To give this model more flexibility, we allowed the decision threshold of the second decision process to be lower than the decision threshold of the first decision process. The second decision threshold was an additional free model parameter. The MATLAB function OU_2D_ 3B_1D_2B_SIM_SC.M, which has been used for evaluating this model, is also part of SIMT. 
Finally, we considered a model that also starts a second decision process when the first threshold crossing occurs, but it does not wait for a second threshold crossing. The second decision process unfolds for a fixed amount of time and is then read out. The sign of the current state of the integrated evidence (of the $1 \mathrm{D}$ process) determines the second choice. The MATLAB function OU_2D_3B_1D_FIXED_TIME_SIM_SC.M, which was used for evaluating this model, is also part of SIMT.

Model fit. The model parameters were identified by an optimization procedure based on the mean RTs. A combination of a global pattern search (provided by MATLAB's Global Optimization Toolbox) and a multidimensional simplex algorithm (provided by MATLAB's Optimization Toolbox) was used to minimize the sum of the squared differences between the mean RTs in the data and the mean RTs predicted by the model, taking the SEs of the estimated means into account. We used the mean RTs for each combination of coherences, regardless of choice (15 data points). For the model, these were obtained by calculating a weighted sum of the predicted mean RTs for the different choices based on the predicted probabilities of these choices.

\section{Results}

We used 11,060 valid decision trials from 7 subjects for analysis and modeling. The overall accuracy of the primary choice was $72 \%$ (chance level would be $33 \%$ for a 3 -alternative forced choice task), which provided us with 7951 correct trials and 3109 error trials for further analysis. How the primary choice and the associated RT depended on the presented stimulus was similar to what we had reported in Niwa and Ditterich (2008) and will be presented in the context of a computational model below.

\section{Second guesses in perceptual decision-making are informed by sensory evidence}

To test whether subjects are able to make an informed second guess, we analyzed the error trials and quantified how often subjects reported what would have been the correct choice as their second guess. If subjects just guessed randomly, this should not deviate significantly from chance (50\%). The correct option, however, was reported as the second guess in $63 \%$ of the error trials, which is highly significantly above chance $\left(p<10^{-6}\right.$; binomial test). This indicates that the second guess was clearly informed by the sensory evidence provided by the motion stimulus.

\section{A computational model that can explain primary choices and associated RTs}

To gain more insight into what information the second guesses were based on, we resorted to computational modeling. In Niwa and Ditterich (2008), we presented an integration-to-threshold model that was able to explain the distribution of choices in the 3 choice multicomponent random-dot motion direction discrimination task as well as the associated RTs. Briefly, in a stochastic process, we modeled three pools of motion-sensitive neurons (for each of the possible directions). Each of these pools had a strong linear response to coherent motion in its preferred direction, a weak linear response to the randomly moving dots in the stimulus, and divisive normalization based on how much coherent motion the stimulus contained driving the other pools. The variance of each pool's output scaled linearly with its mean. The net sensory evidence for each direction, calculated as the difference between one pool's activity and the average activity of the other two, was then fed into an integrator, one for each possible choice. Whichever integrator reached a constant decision threshold first determined the choice, and the time of crossing the decision threshold the decision time. RT was modeled as the sum of the decision time and a fixed residual time, capturing the time needed for aspects of the task other than the decision itself (e.g., initiating an eye movement for reporting the choice). The structure of the model is shown in Figure 2. Further details can be found in Materials and Methods.

If adding the secondary task of reporting a second guess did not alter the way subjects made their primary choice, the same model should still be able to capture the primary choice data and associated RTs from this experiment. To test this, we fitted the model ( 5 free parameters) to the mean RT data. The result of this fit is shown in Figure 3. Filled circles represent the data (with 95\% confidence intervals (CIs), calculated according to the method proposed by Goodman, 1965); lines reflect the model. The motion strength of the strongest motion component in the stimulus is plotted on the horizontal axis; the color of symbols/ lines represents the strength of the other two motion components. The model clearly captures the structure of the mean RT data. If the model were perfect, at least $95 \%$ of the evaluated model mean RTs would be expected to be within the 95\% CIs associated with the data. Our model is close to that: 13 of the 15 mean RTs (87\%) are inside; the two that are outside are still close to the CIs. The estimated model parameters are summarized in Table 2 .

To further test whether the model can explain the primary choice data, we compared the model's prediction for the distribution of primary choices with the actual distribution from the experiment (Fig. 4). These data have not been used yet because the model had only been fitted to mean RT data. The plotting 


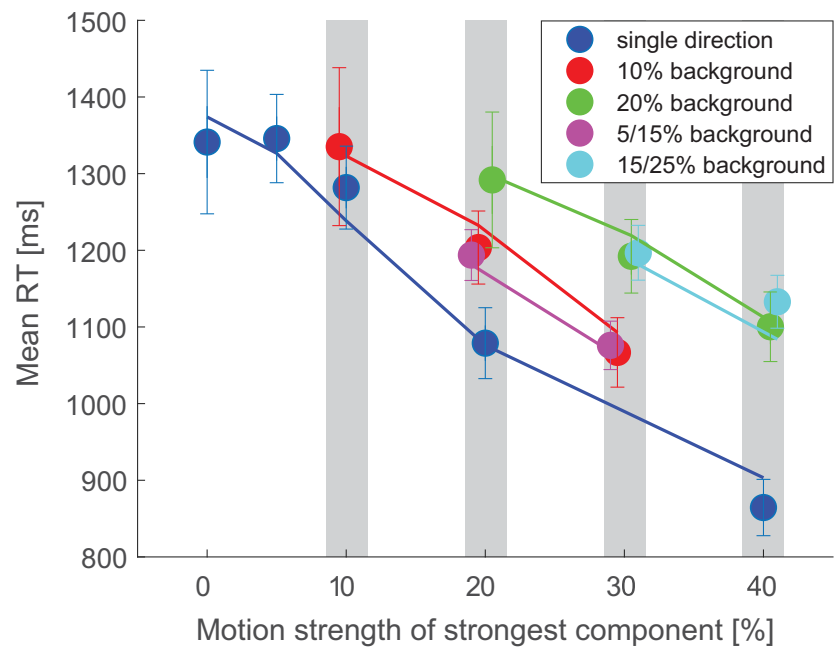

Figure 3. Mean RT data and fitted model. Symbols represent the measured mean RTs for all unique combinations of motion strengths. The motion strength of the strongest component is plotted on the horizontal axis. Colors represent the motion strengths of the two weaker motion components. (For example, the cyan point at 40\% motion strength indicates the mean RT for stimuli with the three motion components having strengths of 40\%, $25 \%$, and $15 \%$, respectively.) Some points have been shifted slightly horizontally to reduce graphical overlap. For example, all points within the gray bar centered on $20 \%$ have a strength of the strongest motion component of exactly $20 \%$. Error bars indicate $95 \%$ Cls. The lines connect the mean RTs from the computational model.

Table 2. Best-fitting model parameters

\begin{tabular}{lc}
\hline Model parameters & Parameter values \\
\hline$g$ & 0.0103 \\
$k_{n}$ & 0.197 \\
$k_{s}$ & 0.616 \\
$k_{v}$ & 0.329 \\
Residual time (ms) & 663 \\
\hline
\end{tabular}

conventions are similar to Figure 3. Circles represent correct choices, squares represent choices of the direction that had intermediate support, and diamonds represent choices of the direction that had the weakest support. A perfect model would predict probabilities, at least $95 \%$ of which would be expected to be within the $95 \%$ CIs associated with the data. While our model is not perfect, 18 of the 23 probabilities $(78 \%)$ are inside, the five that are outside are still close to the CIs. The good agreement between data and model predictions indicates that the model introduced in Niwa and Ditterich (2008) is still able to explain the primary choice data and associated RTs from the current experiment. Thus, asking subjects to report a second guess apparently did not alter the structure of the decision process.

\section{The same computational model can also explain second guesses}

We have demonstrated earlier that subjects can produce informed second guesses when making perceptual decisions between multiple alternatives, but can we gain insight into what governs these second guesses? The idea behind the highly successful integration-to-threshold models in perceptual decisionmaking is that decision-makers accumulate sensory evidence for each of the possible choices until the accumulated evidence for one of them exceeds a decision threshold. How could a subject make an informed second guess in this framework? Assume the decision-maker had access to the states of the integrators that did not win the race at the time of the threshold crossing. What should the distribution of second guesses look like if subjects reported the integrator with the overall second-highest accumulated evidence as their second guess, or, equivalently, the option with the larger accumulated evidence out of the two remaining ones? We took the model, which had been fitted to the mean RTs associated with the primary choice and was able to explain the distribution of primary choices, and obtained the expected distributions of second guesses based on the overall second-highest accumulated evidence.

A comparison between the predicted distributions of second guesses and the actual data on correct trials is shown in Figure 5. Symbols again represent the data (and 95\% CIs); lines reflect the model predictions. On correct trials, by definition, subjects have already reported the correct option as their primary choice. The correct option is therefore no longer available as a second guess. The relative frequency of reporting the correct option as the second guess (filled circles) has to be zero. The only interesting cases are those where the two weaker motion components had different motion strengths (purple and cyan). Squares represent how often subjects reported the direction with the intermediate motion strength as their second guess; diamonds represent how often the weakest motion component was reported.

The same comparison, but now for error trials, is shown in Figure 6. In this case, the correct option can be reported as the second guess, and we had already seen earlier that, across experimental conditions, it was chosen more frequently than chance. The figure shows this relative frequency broken down by experimental condition (circles), adds the relative frequencies of reporting each incorrect option as the second guess (squares and diamonds), and provides the model predictions for comparison. Why, in contrast to the plots we had seen so far, are the squares below the diamonds in this figure? When reporting their primary choice, subjects were more likely to make an error in favor of the motion component with intermediate support rather than picking the weakest component (Fig. 4, squares and diamonds). The weakest component (diamonds) was therefore available as an option for the second guess in substantially more error trials than the component with the intermediate support (squares), which explains why it was overall chosen more frequently. And why does the probability of reporting the direction of the strongest motion component (blue circles/line) not keep increasing monotonically as a function of motion strength? To make an error in a trial with only a single-motion component with $40 \%$ coherence in the first place, the accumulated evidence for this direction has to be unusually low. As a consequence, since the second guess is based on the same accumulated evidence, there is also not a sufficient amount of evidence to support choosing this direction as the second guess. A perfect model would again predict probabilities, at least $95 \%$ of which would be expected to be within the $95 \%$ CIs associated with the data. Across both correct and error trials and not counting the zero-probability events, 26 of the 31 predicted probabilities (84\%) are inside, and the five that are outside are still pretty close to the CIs. Thus, there is good agreement between the data and model predictions, indicating that the reported second guesses are consistent with the idea that the decision-maker has access to information about how much sensory evidence had been accumulated for competing unchosen options at the time when sufficient evidence had been collected to commit to a primary choice.

In summary, a computational model with only five free parameters can account for 15 mean RTs, 16 relative frequencies for the primary choice (not counting the 7 trivial cases of uniform choice distributions when all motion components are 
equally strong and the relative frequency of choosing the third option having to be 1 minus the sum of the relative frequencies of choosing the first or the second option), and 18 relative frequencies for the second guess, again excluding the trivial cases. This strongly suggests that the primary choice and the second guess are produced by a common integration-tothreshold decision process.

\section{Second guesses are best explained by the states of the integrators shortly after threshold crossing}

While the motion stimulus disappeared from the screen when the saccade for reporting the primary choice was detected, there is a delay between the decision threshold crossing and the saccade onset, and some stimulus information is also still in the visual cortical processing pipeline. In the decision confidence literature, it has been proposed that the decision confidence, which is usually reported after the choice, could be informed by sensory evidence that is processed after the choice has been made (Pleskac and Busemeyer, 2010; Moran et al., 2015). To determine whether additional sensory evidence might have contributed to the reported second guesses in our experiment, we created a variant of the model, where the evidence accumulation was allowed to continue for a fixed period of time after the decision threshold had been crossed, before the nonwinning integrators were read out to determine the second choice. Figure $7 A$ shows the deviation between predicted and observed second guesses as a function of the additional integration time. Since the calculated points (blue circles) are simulation-based and therefore slightly noisy, we added a robust polynomial interpolation (solid black line). The best match between predicted and observed second guesses (discrepancy of 0.027 ) is obtained for an additional integration time of $40 \mathrm{~ms}$ (dashed vertical line), that is, when the integrators are read out shortly after the threshold crossing. The discrepancy clearly increases for longer additional integration times. Thus, the second guesses seem to be affected by a small amount of sensory evidence that is processed after the primary choice has been determined, but still largely rely on the same information, as typical decision times in our experiment are an order of magnitude larger. The predicted relative

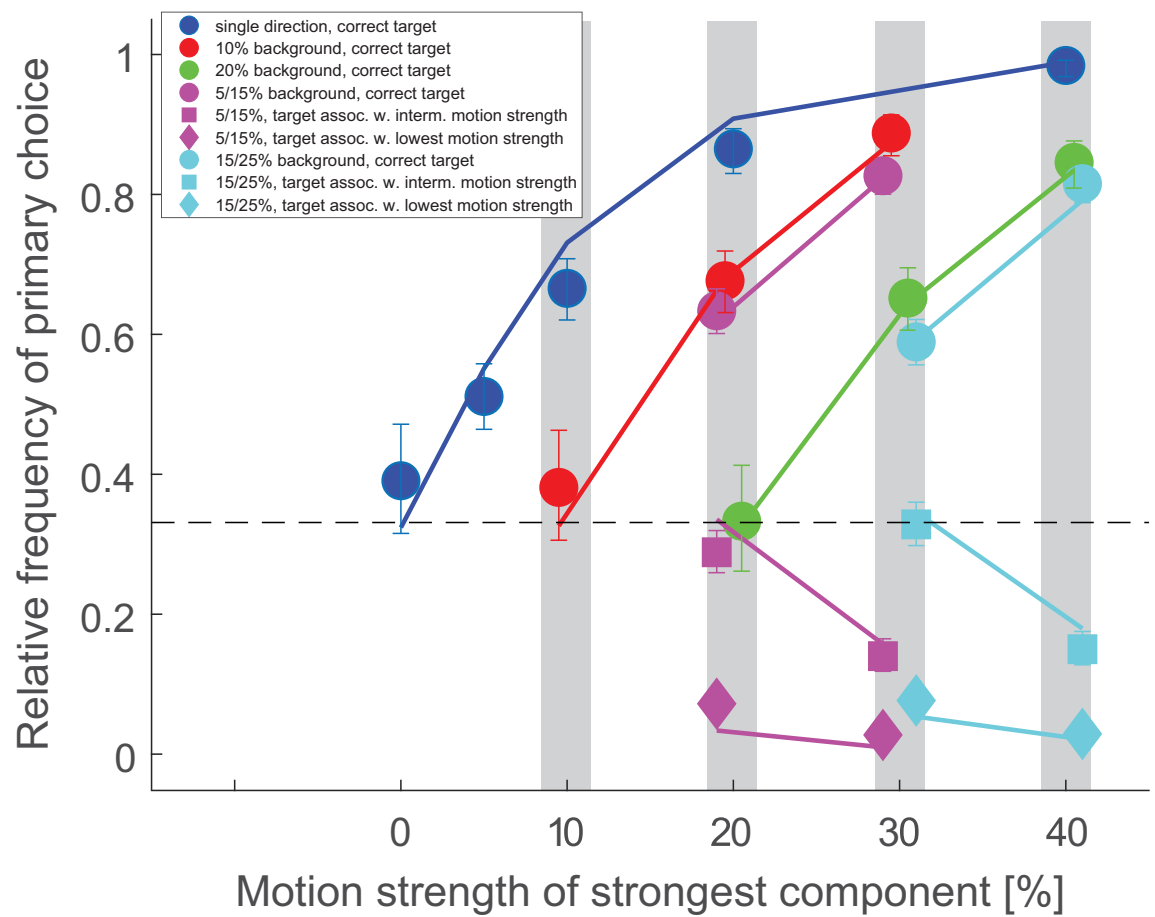

Figure 4. Comparison between the relative frequencies of primary choices and model predictions. Symbols again reflect the data, with error bars indicating $95 \%$ Cls. The lines connect the relative frequencies predicted by the computational model. Circles represent choices of the target associated with the strongest motion component (correct primary choices). Squares represent choosing the target associated with the component with intermediate motion strength. Diamonds represent choosing the target associated with the weakest motion component. Other conventions as in Figure 3. Dashed line indicates chance performance.

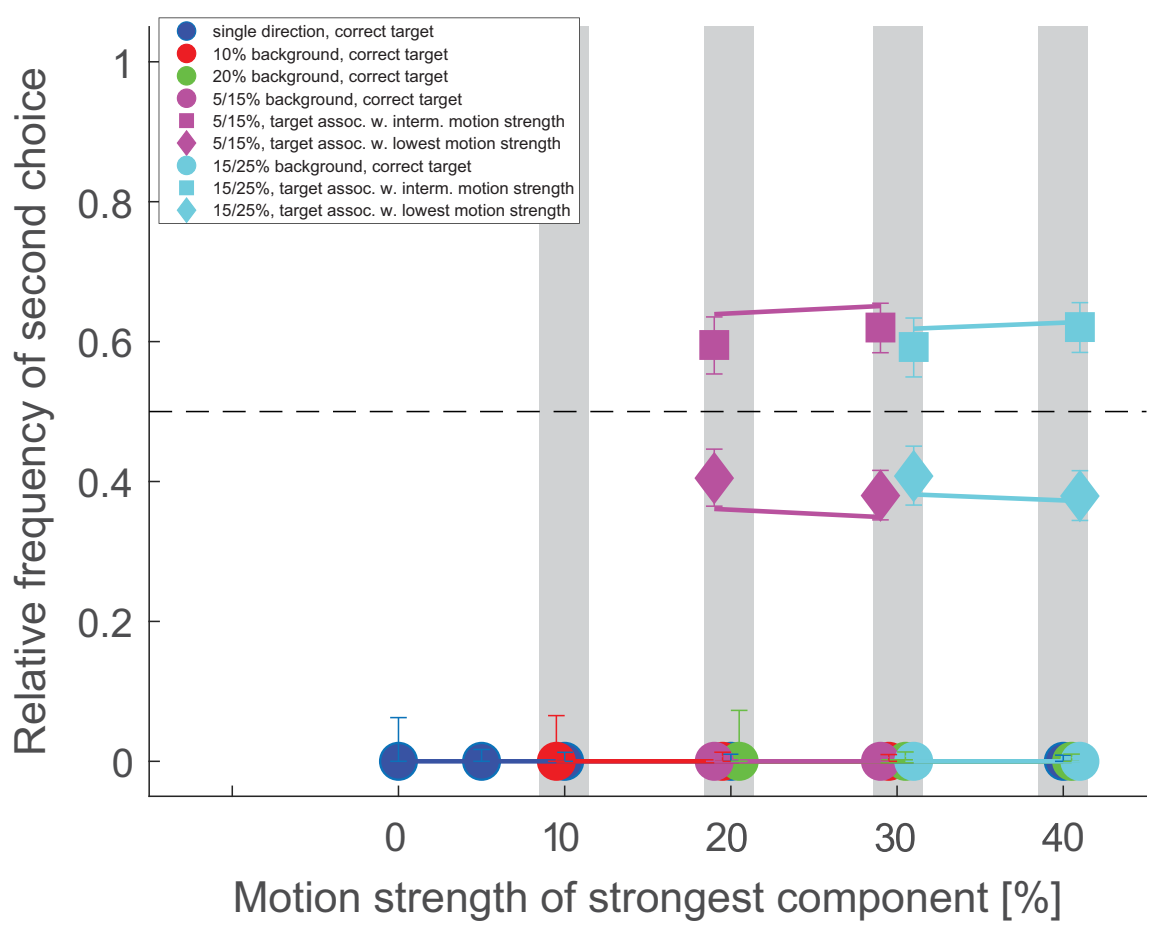

Figure 5. Comparison between relative frequencies of second guesses on correct trials (symbols, with error bars indicating 95\% (ls) and model predictions (lines). Conventions as in Figure 4. On correct trials, the target associated with the strongest motion component has been reported as the primary choice and is not available for the second guess. Therefore, all circles are located at a relative frequency of zero. 


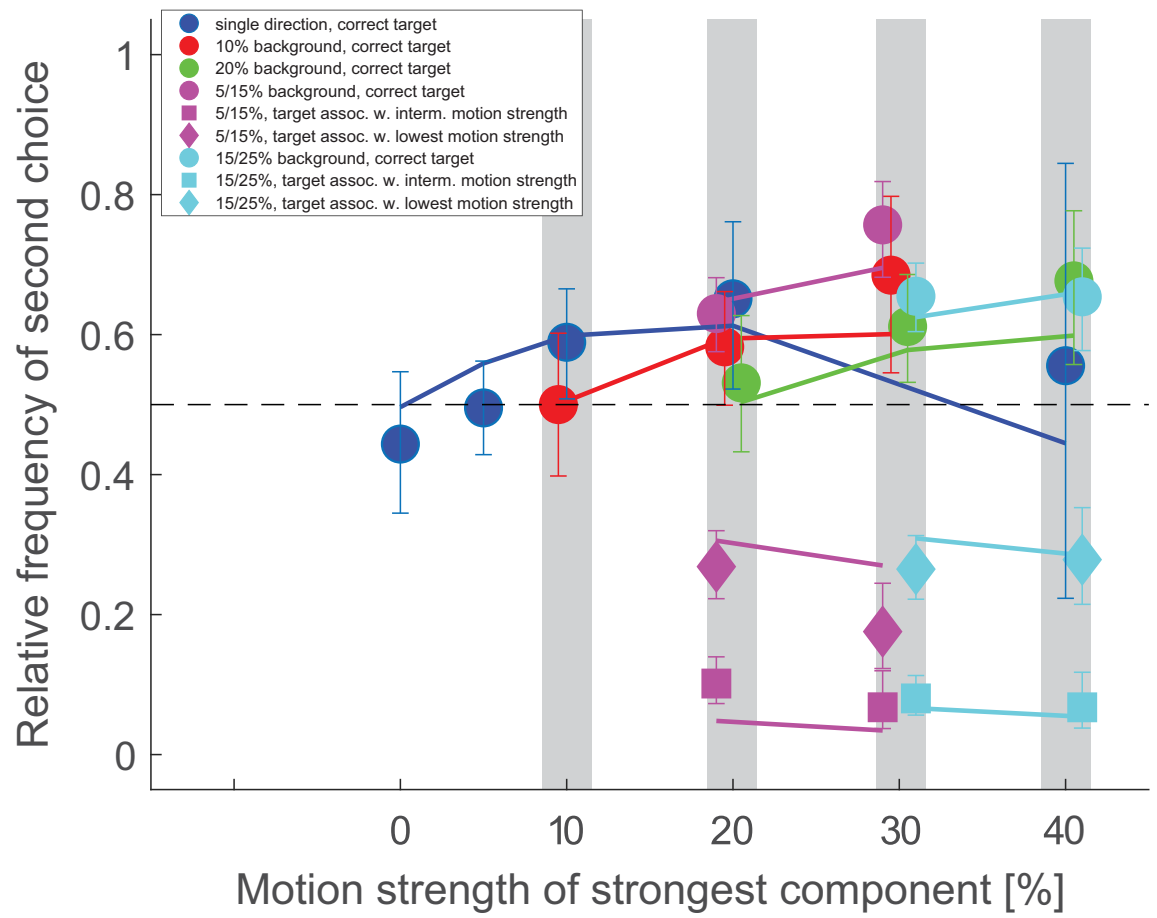

Figure 6. Comparison between relative frequencies of second guesses on error trials (symbols, with error bars indicating $95 \%$ Cls) and model predictions (lines). Conventions as in Figure 5. How often the correct target (circles) was reported as the second choice varied across experimental conditions but was overall significantly above chance (63\%).

frequencies of second guesses for a model with $40 \mathrm{~ms}$ of additional integration time are shown in Figure $7 B, C$. There is no major qualitative difference between these plots and Figures 5 and 6; the match between model predictions (lines) and data (symbols) is just slightly better.

\section{A model waiting for the same decision process to cross a second threshold can be ruled out}

To determine whether the second guesses could also be explained by alternative mechanisms that do not require reading out and comparing the accumulated evidence for the options that did not win the race to threshold, we considered several alternative models. First, we evaluated the possibility that the decision process could continue after the first threshold crossing until a second (different) threshold is crossed. The first threshold crossing would determine the primary choice; the second threshold crossing the second choice and the decision time. One can imagine that in situations where there is much stronger evidence for one particular choice compared with the other alternatives, such a second threshold crossing is unlikely to occur within a reasonable amount of time, in particular when the integration is perfect, and the decision bounds are fixed. We therefore also considered mechanisms with leaky integration and collapsing decision bounds (Ditterich, 2006). It turns out, however, that this class of models, even in the presence of leaky integration and collapsing bounds, makes one key qualitative prediction: decision times should increase, rather than decrease, when the evidence gets stronger. As a consequence, the best mean RT fit that can be obtained is largely flat as a function of motion strength, and the remaining error is $\sim 6$ times as large as the one for the fit shown in Figure 3. Figure $8 \mathrm{~A}$ shows this fitting attempt. This class of models can therefore be ruled out as an alternative explanation.
A model based on a second
integration-to-threshold process
for determining the second choice
makes less accurate predictions for
the distribution of second guesses
We also considered the possibility
that, as soon as the first threshold
crossing occurs, a new decision pro-
cess, only as a 2 AFC between the two
remaining options, is started. A
threshold crossing of the second de-
cision process would then determine
the second choice and the decision
time. When enforcing the same deci-
sion threshold as in the primary de-
cision process, the remaining error
after the mean RT fit is more than an
order of magnitude larger than the
one for the fit shown in Figure 3 . We
therefore considered the possibility
that the decision threshold for the
second decision process could be
lower. The mean RT fit reveals that
the threshold would have to be very
close to zero to be able to account for
the pattern of RTs. A fit with a deci-
sion threshold of 0.052 (compared
with 1 in the case of the first decision
process) resulted in a remaining
error that was only slightly larger than the one for the fit shown in Figure 3. We therefore determined the predicted second guesses for this model (shown in Fig. 8B,C). The discrepancy between predicted and observed second guesses, following the same convention as the one used in Figure $7 A$, was 0.129 (Fig. $8 D$, red dashed line), about 5 times as big as the one for the model shown in Figure $7 B, C$. Thus, this model also cannot capture the data pattern as well as our original model.

A model based on a second, fixed-duration decision process for determining the second choice provides the second-best explanation for the distribution of second guesses

As a final possibility, we considered that the second decision process might not be terminated by a threshold crossing, but rather end after a fixed amount of time. The process would be read out at that point, and the sign of the accumulated evidence would determine the second choice. The discrepancy between predicted and observed second guesses for this model, as a function of the duration of the second decision process, is shown in Figure $8 D$. Since the calculated points (blue circles) are simulation-based and therefore slightly noisy, we again added a robust polynomial interpolation (solid blue line). The best match is observed for an integration time of $70 \mathrm{~ms}$, but the discrepancy is still $0.081, \sim 3$ times as big as the one for the model shown in Figure $7 B, C$ (Fig. $8 D$, solid black). This model's predictions for the second guesses are shown in Figure $8 E, F$. In contrast to our original model, which predicted the nonmonotonic relationship between motion strength and the probability of choosing the strongest motion component as the second guess on error trials (Fig. $8 F$, blue circles), this model predicts a monotonic relationship (blue line). This difference results from the fresh start of evidence accumulation in the second decision process, rather than the second guess being substantially affected by the accumulated evidence that led 
to the primary choice. Since $70 \mathrm{~ms}$ is needed for the second integration process, the residual time would be reduced to $593 \mathrm{~ms}$ in this case. While this model provides the second-best explanation, our original model still provides the better explanation for the observed pattern of second guesses.

\section{Discussion}

We asked human subjects to make a perceptual decision among three alternatives and to report not only their primary choice, but also a second guess. Our data indicate that this second guess is not random, but clearly informed by the sensory evidence. A single integration-to-threshold model cannot only explain the distribution of primary choices and the associated RTs, but also the distribution of second guesses. This suggests that the second guess is generated based on largely the same accumulated evidence that is also used to produce the primary choice. The second guess appears to be governed by the ranking of the amounts of evidence that have been accumulated by the integrators that did not win the race to threshold, which are apparently accessible.

We also considered alternative models. The only other model that was able to largely capture the data pattern, although not as well as the model based on reading out the states of the integrators that had not crossed the decision threshold yet shortly after the winning integrator crossing its threshold, was a model based on starting a new decision process when the threshold crossing determining the primary choice occurred. The process had to be set up as a decision between the remaining alternatives and read out after a fixed amount of time ( $\sim 70 \mathrm{~ms})$.

Relationship with decision confidence Human subjects cannot only report their choice when making a perceptual decision, but also express a level of confidence in their decision. A substantial body of literature has been devoted to how well calibrated this decision confidence is and how it might be computed. Ideally, the level of confidence should match the accuracy of the decision. However, this is typically not the case, and human subjects have been reported to be either underconfident or overconfident, depending on the difficulty of the decision (for review, see Rahnev and Denison, 2018). Confidence clearly is informed by the available sensory evidence, but how? Vickers
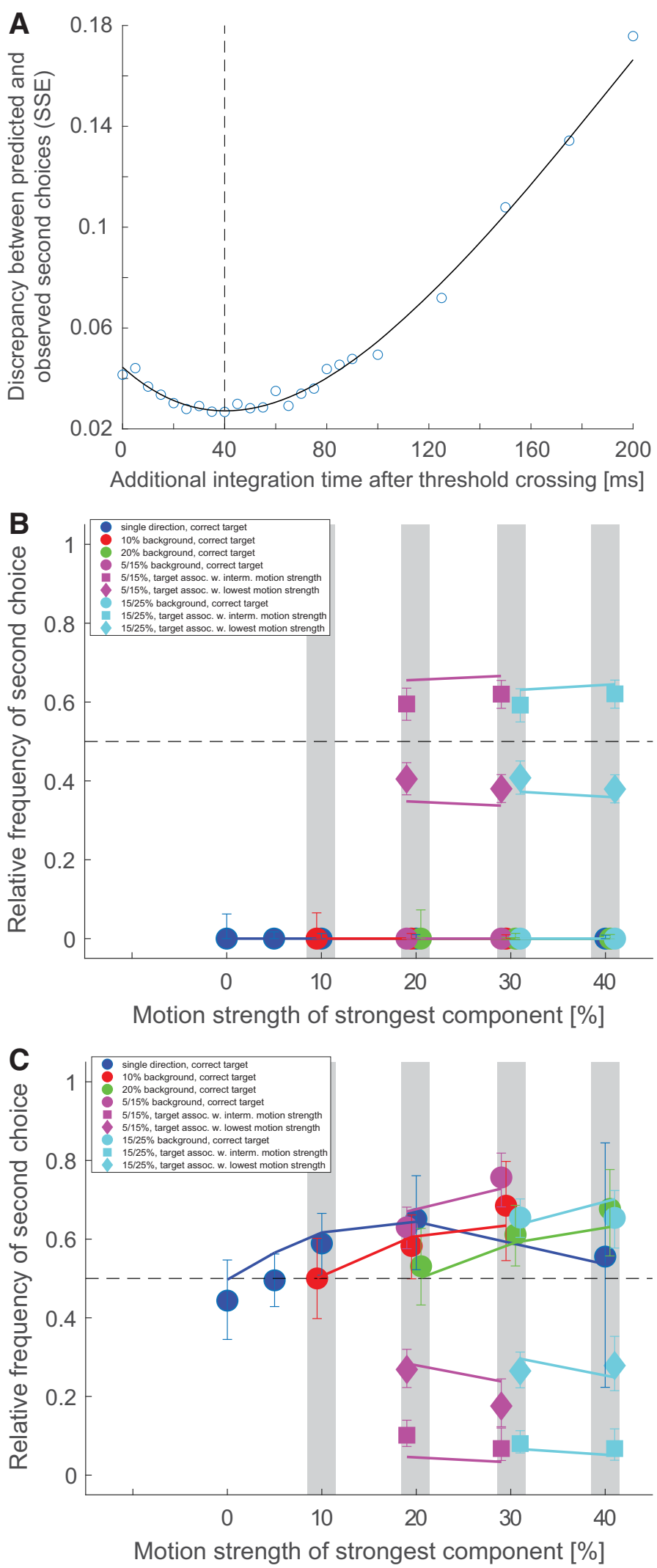

Figure 7. Predictions for second guesses when integration is allowed to continue after the threshold crossing. $\boldsymbol{A}$, Discrepancy between predicted and observed second guesses as a function of additional integration time before the accumulated evidence is read out. A minimum (best match) is observed at $40 \mathrm{~ms}$. $\boldsymbol{B}$, Predicted second guesses on correct trials with $40 \mathrm{~ms}$ additional integration time (same format as in Fig. 5). C, Predicted second guesses on error trials with 40 ms additional integration time (same format as in Fig. 6). 

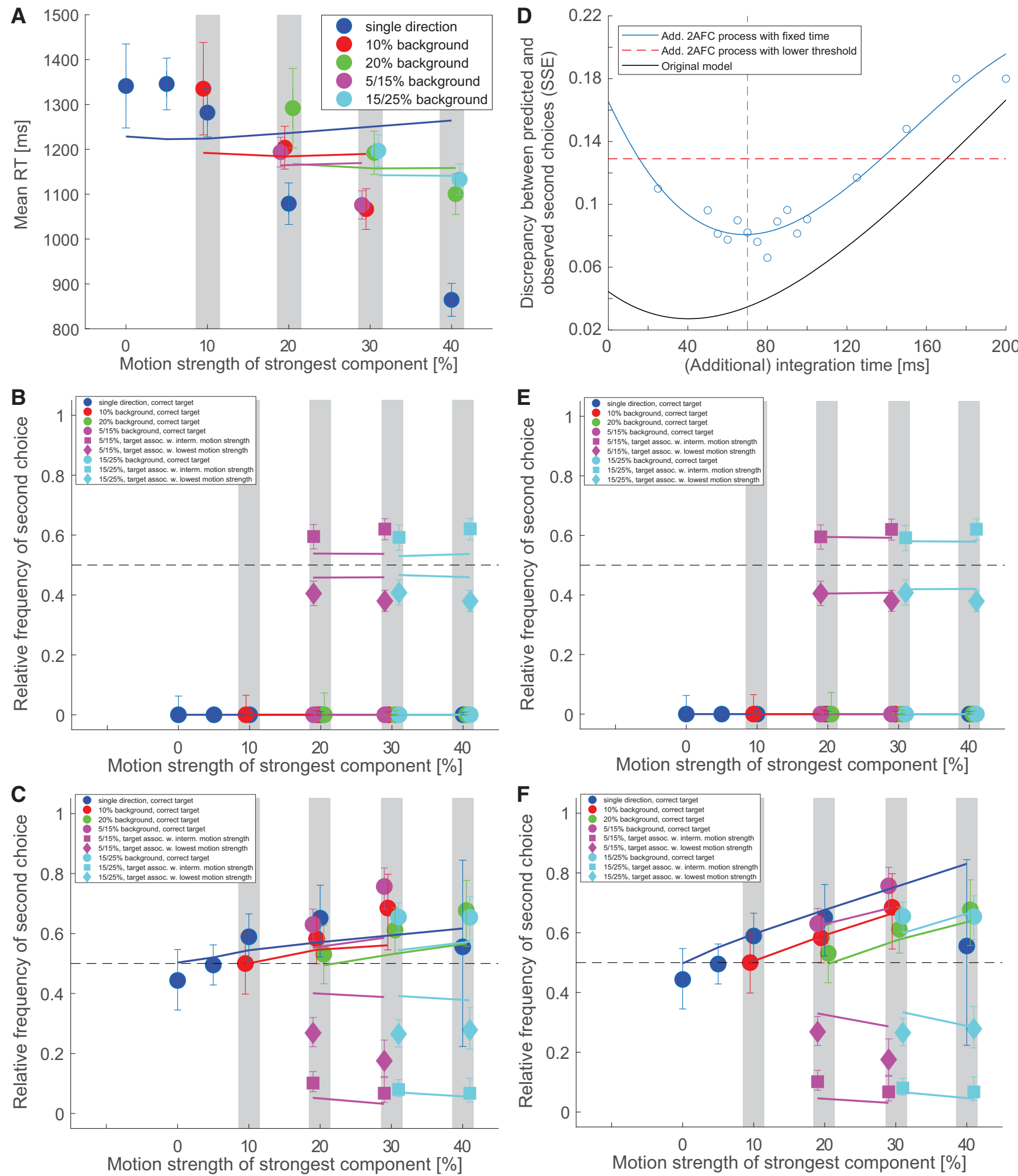

Figure 8. Alternative models. $\boldsymbol{A}$, Mean RT fit for a model that waits for a second threshold crossing, but allowing leaky integration and collapsing bounds (same format as in Fig. 3). $\boldsymbol{B}$, Predicted second guesses on correct trials for a model that starts a new 2AFC decision process to determine the second choice and waits for a threshold crossing, but allowing a lower threshold than in the primary decision process (same format as in Fig. 5). $\boldsymbol{C}$, Same as in $\boldsymbol{B}$, but for error trials (same format as in Fig. 6). $\boldsymbol{D}$, Discrepancy between predicted and observed second guesses as a function of integration time for a model that starts a new 2AFC decision process to determine the second choice and reads the process out after a fixed amount of time (blue). A minimum (best match) is observed at $70 \mathrm{~ms}$. For comparison, the curve for the original model (black) and the value for the model with a low threshold (red) are also shown. $E$, Predicted second guesses on correct trials for a model that starts a new 2AFC decision process to determine the second choice and integrates the sensory evidence for $70 \mathrm{~ms}$ before the process is read out (same format as in Fig. 5). $\boldsymbol{F}$, Same as in $\boldsymbol{E}$, but for error trials (same format as in Fig. 6). 
(1979) suggested that it depends on the balance of evidence. The more dissimilar the amounts of evidence in favor of the available options are at the time of making a decision, the more confident the observer can be about the choice. This information can be extracted from the decision process itself. While the idea is incompatible with the popular 1D drift-diffusion model for 2AFCs, which is equivalent to a race between two accumulators that receive perfectly anticorrelated instantaneous net evidence and, as a consequence, always has the losing integrator in an identical state when the winning integrator exceeds the decision threshold, it can be applied to alternative models. For example, Ditterich (2006) demonstrated that a model based on partially anticorrelated accumulators provides a better account of decision-related activity in the parietal association cortex of monkeys performing a perceptual decision task. Neurons coding for the losing alternative do not show a stereotyped activity level when the neurons coding for the winning alternative reach threshold. This information could be used to inform confidence. MorenoBote (2010) formalized how confidence can be extracted from diffusion models with partially correlated integrators. An alternative mechanism was proposed by Smith and Vickers (1988). According to their model, only one of the integrators is updated at a particular time, the one receiving positive instantaneous net sensory evidence, which also results in the losing accumulator being in different states when the winning accumulator reaches threshold.

Gaining neurophysiological insights into the neural mechanism underlying decision confidence from animal experiments is challenging, as animals cannot be asked directly to provide an explicit confidence rating. However, animal tasks have been developed, which require the animal to produce a behavior that should be informed by decision confidence (for review, see Hanks and Summerfield, 2017). For example, Kiani and Shadlen (2009) trained monkeys to make a perceptual decision between two alternatives. In a random subset of trials, the researchers offered a third option, a sure bet resulting in a smaller, but certain reward, whereas the animals could gain a larger reward if they engaged in a choice and reported the correct option. The animals were more likely to choose the sure bet the weaker the sensory evidence (motion coherence) was and the shorter they were allowed to watch the motion stimulus. Importantly, decision-related neurons in parietal association cortex that have the signature of carrying accumulated evidence showed either strong or weak activation when the animal engaged in a choice, but intermediate activation when opting for the sure bet, suggesting that the information encoded in these neurons does not only govern choice, but also inform confidence. The study further suggested that decision confidence does not only depend on accumulated evidence, but also on elapsed time, which was confirmed explicitly in a later human psychophysics experiment (Kiani et al., 2014) and is also formalized in the Moreno-Bote (2010) model. Animal experiments on decision confidence have received some criticism, primarily claiming that the tasks could potentially be solved without requiring any meta-cognition, for example, by treating tasks with a sure bet as a multialternative decision task (Insabato et al., 2016, 2017). However, Kepecs and Mainen (2012) pointed out that the same scrutiny should then also be applied to human tasks.

The view that confidence is governed by the same information that determines the choice and, in particular, by the balance of evidence has been challenged by experiments that found that confidence primarily relies on response-congruent evidence (Zylberberg et al., 2012; Maniscalco et al., 2016). The authors reported that, while choices in their experiments were governed by the balance of evidence, confidence was primarily determined by the amount of evidence for the chosen option and largely insensitive to the amount of evidence for the nonchosen alternative. Dual-stage or second-order models are also at odds with the idea that choice and confidence rely on the same information (Pleskac and Busemeyer, 2010; Moran et al., 2015; Fleming and Daw, 2017). These models posit that confidence ratings rely on a postdecision process that is informed by the outcome of the decision process, but not exclusively.

Different studies have therefore found the information on which choice and decision confidence are based to overlap to varying degrees. We have addressed a similar question for the mechanism underlying second guesses. Our results indicate that the distribution of second guesses is most compatible with a decision mechanism that largely uses the same accumulated evidence for determining both the primary and the second choice. We found the best match between model predictions and data, when the decision process was allowed to continue for a very short period of time (compared with typical decision times in our experiment), $\sim 40 \mathrm{~ms}$, after the threshold crossing determining the primary choice, before the states of the remaining integrators are read out to determine the second choice.

\section{Second guessing in other cognitive functions}

In 1961, Signal Detection Theory was still in its infancy and competing with the prevailing "high threshold" model of sensory perception, Swets et al. (1961) published a paper proposing that a second-choice paradigm in multi-interval signal detection could help distinguish between the competing ideas. However, secondchoice paradigms have not been pursued further in the area of perceptual decision-making, in particular not since the field has turned to sequential sampling models to explain not only choices, but also decision times. Instead, the proposal by Swets et al. (1961) got picked up in the memory literature, there typically referred to as a $4 \mathrm{AFC}-2 \mathrm{R}$ (four-alternative forced choice with two responses) paradigm, as the field was also debating whether recognition memory was best described by a threshold process or by a continuous memory strength process. Parks and Yonelinas (2009) used a second-choice paradigm to gather experimental evidence beyond the receiver operating characteristic analysis that the field had relied on previously. Kellen and Klauer (2011) followed up with a more detailed model-based analysis. Earlier, second guesses had already been used to study mechanisms underlying the effect of misinformation on memory recall (Wright et al., 1996). More recently, second guesses have also been used to study conflict detection mechanisms in reasoning (Bago et al., 2019).

\section{Second guesses as a tool for studying knowledge about the decision process}

We have shown that human subjects can produce informed second guesses when making perceptual decisions between multiple alternatives and that these second choices follow a distribution that would be expected if they were governed by the relative amounts of accumulated net sensory evidence for each option at the time of the largest accumulated evidence reaching a bound. Second-choice paradigms therefore cannot only be used in the context of Signal Detection Theory, as they have in the past, but also with accumulation-of-evidence frameworks. In addition to decision confidence, the study of second guesses provides another useful tool for gaining insight into the decision process and what information a decision-maker has access to about the outcome of a decision, beyond the discrete choice. Similar to the 
neurophysiological work on decision confidence, we expect future studies to be able to establish a link between second guesses and underlying neural activity.

\section{References}

Bago B, Raoelison M, De Neys W (2019) Second-guess: testing the specificity of error detection in the bat-and-ball problem. Acta Psychol (Amst) 193:214-228.

Bollimunta A, Totten D, Ditterich J (2012) Neural dynamics of choice: single-trial analysis of decision-related activity in parietal cortex. J Neurosci 32:12684-12701.

Brainard DH (1997) The Psychophysics Toolbox. Spat Vis 10:433-436.

Ditterich J (2006) Stochastic models of decisions about motion direction: behavior and physiology. Neural Netw 19:981-1012.

Ditterich J (2010) A comparison between mechanisms of multi-alternative perceptual decision making: ability to explain human behavior, predictions for neurophysiology, and relationship with decision theory. Front Neurosci 4:184.

Fleming SM, Daw ND (2017) Self-evaluation of decision-making: a general Bayesian framework for metacognitive computation. Psychol Rev 124:91-114.

Forstmann BU, Ratcliff R, Wagenmakers EJ (2016) Sequential sampling models in cognitive neuroscience: advantages, applications, and extensions. Annu Rev Psychol 67:641-666.

Goodman LA (1965) On simultaneous CIs for multinomial proportions. Technometrics 7:247-254.

Hanks TD, Summerfield C (2017) Perceptual decision making in rodents, monkeys, and humans. Neuron 93:15-31.

Insabato A, Pannunzi M, Deco G (2016) Neural correlates of metacognition: a critical perspective on current tasks. Neurosci Biobehav Rev 71:167175.

Insabato A, Pannunzi M, Deco G (2017) Multiple choice neurodynamical model of the uncertain option task. PLoS Comput Biol 13:e1005250.

Kellen D, Klauer KC (2011) Evaluating models of recognition memory using first- and second-choice responses. J Math Psychol 55:251-266.

Kepecs A, Mainen ZF (2012) A computational framework for the study of confidence in humans and animals. Philos Trans R Soc Lond B Biol Sci 367:1322-1337.

Kiani R, Shadlen MN (2009) Representation of confidence associated with a decision by neurons in the parietal cortex. Science 324:759-764.

Kiani R, Corthell L, Shadlen MN (2014) Choice certainty is informed by both evidence and decision time. Neuron 84:1329-1342.

Latimer KW, Yates JL, Meister ML, Huk AC, Pillow JW (2015) Single-trial spike trains in parietal cortex reveal discrete steps during decision-making. Science 349:184-187.
Luce RD (1986) Response times: their role in inferring elementary mental organization. Oxford: Oxford University Press.

Maniscalco B, Peters MA, Lau H (2016) Heuristic use of perceptual evidence leads to dissociation between performance and metacognitive sensitivity. Atten Percept Psychophys 78:923-937.

Moran R, Teodorescu AR, Usher M (2015) Post choice information integration as a causal determinant of confidence: novel data and a computational account. Cogn Psychol 78:99-147.

Moreno-Bote R (2010) Decision confidence and uncertainty in diffusion models with partially correlated neuronal integrators. Neural Comput 22:1786-1811

Niwa M, Ditterich J (2008) Perceptual decisions between multiple directions of visual motion. J Neurosci 28:4435-4445.

Palmer J, Huk AC, Shadlen MN (2005) The effect of stimulus strength on the speed and accuracy of a perceptual decision. J Vis 5:376-404.

Parks CM, Yonelinas AP (2009) Evidence for a memory threshold in secondchoice recognition memory responses. Proc Natl Acad Sci USA 106:11515-11519.

Pelli DG (1997) The VideoToolbox software for visual psychophysics: transforming numbers into movies. Spat Vis 10:437-442.

Pleskac TJ, Busemeyer JR (2010) Two-stage dynamic signal detection: a theory of choice, decision time, and confidence. Psychol Rev 117:864901.

Rahnev D, Denison RN (2018) Suboptimality in perceptual decision making. Behav Brain Sci 41:1-107.

Ratcliff R, Smith PL (2004) A comparison of sequential sampling models for two-choice reaction time. Psychol Rev 111:333-367.

Ratcliff R, McKoon G (2008) The diffusion decision model: theory and data for two-choice decision tasks. Neural Comput 20:873-922.

Roitman JD, Shadlen MN (2002) Response of neurons in the lateral intraparietal area during a combined visual discrimination reaction time task. J Neurosci 22:9475-9489.

Shadlen MN, Newsome WT (2001) Neural basis of a perceptual decision in the parietal cortex (area LIP) of the rhesus monkey. J Neurophysiol 86:1916-1936.

Smith PL, Vickers D (1988) The accumulator model of 2-choice discrimination. J Math Psychol 32:135-168.

Swets J, Tanner WP Jr, Birdsall TG (1961) Decision processes in perception. Psychol Rev 68:301-340.

Treue S, Hol K, Rauber HJ (2000) Seeing multiple directions of motion-physiology and psychophysics. Nat Neurosci 3:270-276.

Vickers D (1979) Decision processes in visual perception. San Diego: Academic.

Wright DB, Varley S, Belton A (1996) Accurate second guesses in misinformation studies. Appl Cogn Psychol 10:13-21.

Zylberberg A, Barttfeld P, Sigman M (2012) The construction of confidence in a perceptual decision. Front Integr Neurosci 6:79. 